\title{
Autoignition Flame Dynamics in Sequential Combustors
}

\section{Conference Paper}

Author(s):

Schulz, O.; Noiray, Nicolas

Publication date:

2016

Permanent link:

https://doi.org/10.3929/ethz-a-010819327

Rights / license:

In Copyright - Non-Commercial Use Permitted 


\title{
Autoignition Flame Dynamics in Sequential Combustors
}

\author{
O. Schulz ${ }^{*, a}$, N. Noiray ${ }^{\mathrm{a}}$ \\ ${ }^{a}$ CAPS Laboratory, Department of Mechanical and Process Engineering, \\ ETH Zurich, 8092 Zurich, Switzerland
}

\begin{abstract}
This numerical study deals with the heat release rate response of a sequential combustor flame to temperature perturbations. These flames are found in the new generation of gas turbine combustors, which are based on sequential combustion technologies. It is shown that the temperature perturbations $T^{\prime}$ upstream of the fuel injection induce mixture fraction oscillations $Z^{\prime}$ which also propagate toward the reaction zone, and that the combination of these two perturbations yields a strongly nonlinear heat release rate response $Q^{\prime}$. Large Eddy Simulation (LES) were performed using an Analytically Reduced Chemistry (ARC) mechanism, which includes 22 transported species in combination with the Dynamic Thickened Flame model (DTF). The burner flame transfer function (BFTF) giving the flame response with respect to $T^{\prime}$ is obtained using a broadbandexcitation-based system identification (SI) as well as harmonic single frequency excitations. A simplified configuration with perfectly premixed conditions is considered to quantify the respective contributions of the different types of perturbation. These simulations highlight (i) the strong nonlinearity of the flame response to temperature perturbations, and (ii) the difficulty to break down the global flame response into independent driving mechanisms by making use of "simplified" configurations.
\end{abstract}

\section{Introduction}

The demand for pollutant emission reduction, higher efficiency, increased operational and fuel flexibility pushes the research and development of novel gas turbine combustor architectures forward. An important technology step change is the development of constant pressure sequential combustion systems, also known as axial staging concepts $[11,18,21]$. In these combustors, the first stage is based on lean premixed turbulent combustion, with swirled flames anchored on conventional burners. Their thermoacoustic dynamics has been investigated in countless studies, e.g. [5, 7, 26, 9, 16].

Recently, an increasing number of studies dealing with the combustion process taking place in the second stage have been published. The second stage fuel is injected into the vitiated hot-gas flowing from the first stage, leading to a reaction zone stabilised by mixture autoignition and flame propagation. One can refer to previous numerical studies $[19,29,27]$ and experimental work $[27,25,1]$.

The thermoacoustic stability is one of the main challenges in gas turbine combustor engineering, especially in lean conditions [14]. Thermoacoustic instabilities can result from a constructive interaction between the acoustic pressure and unsteady heat release rates from the flame [20], which can lead to high level acoustic pressure oscillations in the combustor [14].

Coherent oscillations of the flame heat release rate can be generated by various perturbations, such as acoustic velocity [28], acoustically triggered equivalence ratio fluctuations [3] or acoustically triggered temperature fluctuations. This numerical study investigates the heat release rate response to temperature perturbations of a lean second stage combustion configuration with a cold methane-air jet in vitiated hot cross flow with area expansion allowing flame stabilisation downstream of the fuel injection.

\footnotetext{
* Corresponding author

Email address: oschulz@ethz.ch (O. Schulz)
} 
The link between the temperature perturbations and the heat release rate response is given in terms of the frequency response of the flame or also referred to as the flame transfer function (FTF). A commonly used method to experimentally and numerically obtain the FTF is imposing sinusoidal harmonic excitation upstream of the flame and measuring the corresponding heat release rate response (e.g. [7, 16, 12]). One of the method advantages is, that the non-linear flame response which is characterised by a flame describing function (FDF) can be obtained by measuring the heat release rate response due to excitation with varying oscillation level (e.g. [7]). Nevertheless one disadvantage is the considerable computational cost because many simulations have to be performed to cover the range of excitation frequency and amplitude of interest. A computationally more efficient alternative to extract the FTF is to perform system identification (SI) of the flame response to a broadband excitation, a powerful technique which has been recently reviewed by Polifke [17] (one can also refer for instance to [23]). In the present work, a sequential combustor is treated as a single-input-single-output (SISO) Black Box, with temperature perturbations $T^{\prime}$ at the inlet (input) and heat release rate response $Q^{\prime}$ (output). One of the major advantages of CFD/SI is, that the frequency flame response can be obtained with a single simulation and simulation times are of the order of a few periods of the lowest frequency to be considered [17]. One of the drawbacks is the assumption that for sufficiently small perturbations the CFD simulation approximates a linear, time-invariant (LTI) system. Therefore one can often assume that with low forcing amplitudes the flame responds linearly to incoming perturbations. However temperature fluctuations can result in strong flame movements even for low excitation amplitudes due to the non-linear dependence of the reaction rate on the temperature in the Arrhenius expression, as shown for example by Mastorakos [15].

An example demonstrating the strong influence of the hot-gas temperature on the autoignition position is the well-documented experimental Cabra flame configuration [4]. A cold methane-air jet is injected into hot vitiated co-flow and turbulence chemistry interactions are similar to the ones in a sequential burner. Autoignition is the main stabilisation mechanism, as shown for example by Schulz et al. [22] and the hot-gas temperature is the dominant parameter significantly influencing the liftoff height of the flame as seen for instance in $[4,13]$.

The goal of this study is to determine the heat release rate response to temperature fluctuations of a sequential combustor flame with CFD/SI and harmonic single frequency excitation. The combustor is treated as a Black Box which does not allow to identify the contribution of individual mechanisms to the global flame response for example to optimise the burner design. With the aim to gain further insight into the internal mechanisms of the Black Box, individual subsystems are considered. Therefore burner transfer functions relating temperature perturbation at the domain inlet with (i) temperature fluctuations and (ii) temperature induced equivalence ratio fluctuations both extracted before the flame are obtained. The subsystem flame is studied in a simplified configuration to break down the global flame response into independent driving mechanisms and to determine the start of the non-linear flame response for these different types of perturbations.

\section{Sequential Burner}

A sketch of the sequential burner considered in this study is shown in Figure 1a. A cold methaneair jet $\left(T_{j e t}=320 \mathrm{~K}, u_{\text {jet }}=200 \mathrm{~m} / \mathrm{s}\right)$ is injected into an air-diluted hot-gas cross flow $\left(T_{H G}=1450 \mathrm{~K}\right.$, $\left.u_{H G}=65 \mathrm{~m} / \mathrm{s}\right)$. This cross flow results from the mixture of first stage burner products and additional fresh air. The corresponding upstream flow is not considered in the present study. The fuel injection is acoustically stiff ensuring constant fuel mass flow delivery. The global equivalence ratio is fixed at $\phi=0.65$. The ignition sequence does not necessitate external heat source: The mixture auto-ignites and the recirculation zones are progressively filled with hot products until steady state is reached. This configuration ensures robust anchoring of the flame at the rim of the mixing section outlet. An instantaneous temperature flow field at steady state condition is shown in Fig. $1 \mathrm{~b}$.

Figure 1c shows the Q-criterion iso-contour together with the iso-contour of methane mass fraction $Y_{\mathrm{CH}_{4}}$. Vortices generated by the 2 vortex generators interact with the fuel jet and therefore ensure an improved mixing of the fuel with the air-diluted hot-gas cross flow.

This is a pre-print version. Presented at International Symposium: Thermoacoustic Instabilities in Gas Turbines and Rocket Engines, May 30 - June 02, 2016 Munich, Germany. 


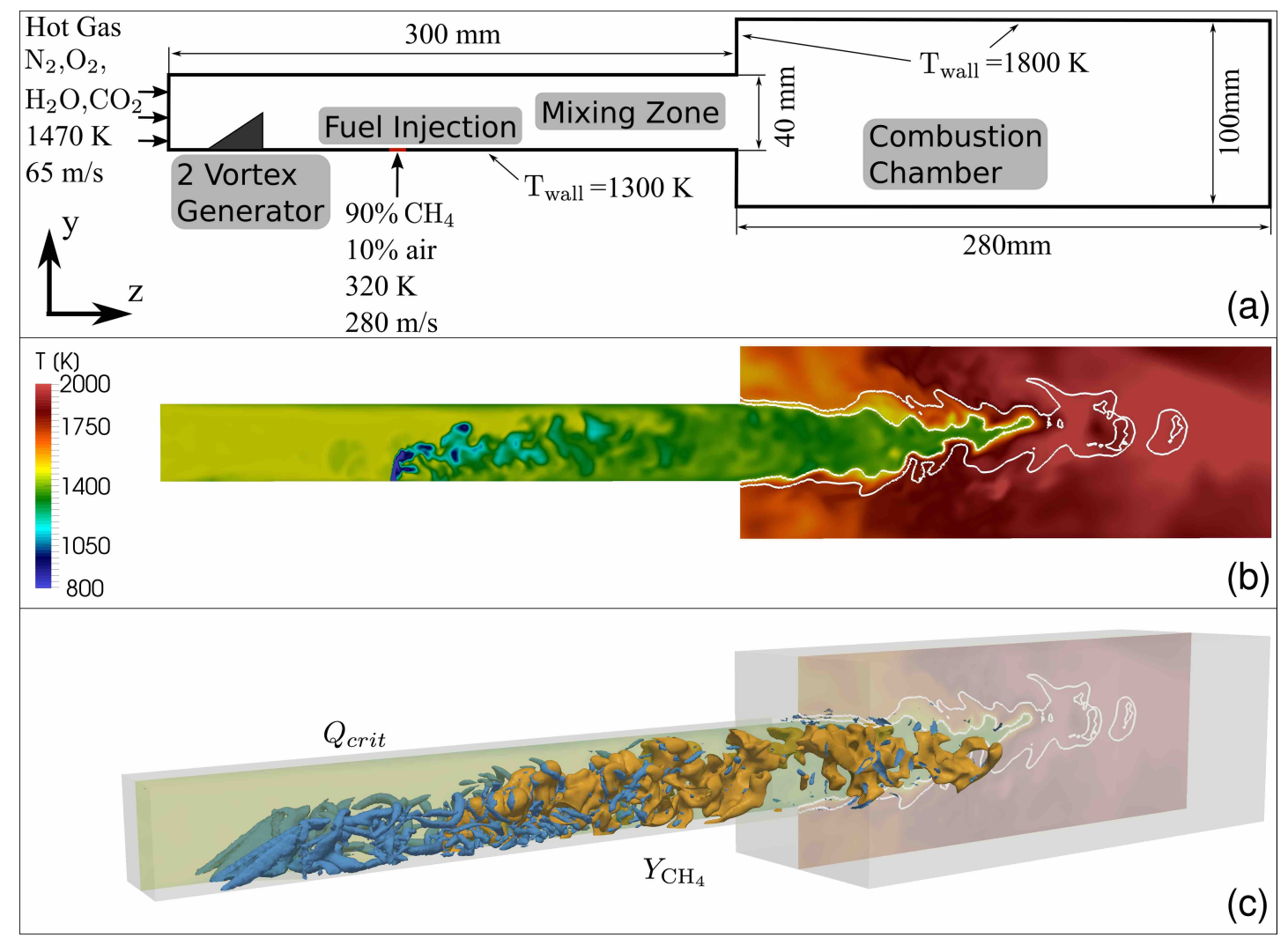

Figure 1: (a) Sketch of the sequential combustor. (b) Representative snapshot of temperature field and heat release rate isoline superimposed in white at $2 \times 10^{8} \mathrm{~W} / \mathrm{m}^{3}$. (c) Flow structures from Q-criterion iso-surface at $4 \times 10^{7}$ in blue and $\mathrm{CH}_{4}$ mass fraction iso-surface at 0.04 in orange. Translucent cut taken from (b). (d) Computational mesh with axial cut through $x=15 \mathrm{~mm}$ and transverse cut through fuel injector.

\section{Numerical Methods and validation case for the chemistry scheme}

The dimensions of the 3-D domain is shown in Fig. 1a. The flow-through time is estimated as $0.58 \mathrm{~m} / u_{H G}=9 \mathrm{~ms}$. The explicit cell-vertex parallel code AVBP [10] was used to perform compressible reactive Large Eddy Simulations (LES) with a space and time accuracy of third order. The subgrid Reynolds stress is modelled by the Smagorinsky approach. Moreover a coupled velocity/temperature wall-model is used allowing cell sizes at the walls which do not resolve the boundary layer. The non-reflecting NavierStokes Characteristic Boundary Conditions formulation is used for the two inlets and the outlet. A heat loss formulation is imposed at the domain walls via $\dot{q}=\left(T_{\text {wall }}-T_{\infty}\right) / R_{w}$, with a reference temperature set to $T_{\infty}=300 \mathrm{~K}$. For the mixing zone the thermal resistance is determined as $R_{w}=0.02 \mathrm{Km}^{2} / \mathrm{W}$ and for the combustion chamber as $R_{w}=0.04 \mathrm{Km}^{2} / \mathrm{W}$. 2-D wall resolved simulations at characteristic velocities and temperatures showed that these $R_{w}$ result into temperatures of $T_{\text {wall }} \approx 700 \mathrm{~K}$ in the mixing zone and $T_{\text {wall }} \approx 1000 \mathrm{~K}$ in the combustion chamber.

The unstructured computational mesh is presented in Fig. 1d, it has a total number of 1.9 million nodes (11.1 million cells). As shown by Prause et al. [19] the mesh size can have a significant influence on the mixing and therefore on the flame position. In order to decrease uncertainties due to insufficient grid resolution a grid sensitivity analysis was performed. The meshes have the following characteristic cell sizes in the mixing zone downstream of the fuel injection: a coarse grid (i) with $\Delta x=1.125 \mathrm{~mm}$, (ii) with $\Delta x=0.9 \mathrm{~mm}$, (iii) with $\Delta x=0.675 \mathrm{~mm}$, (iv) with $\Delta x=0.55 \mathrm{~mm}$ and a very fine grid (v) with $\Delta x=0.5 \mathrm{~mm}$.

Figure 2 shows the time averaged profiles of mixture fraction $Z$ for the five different meshes at the center

This is a pre-print version. Presented at International Symposium: Thermoacoustic Instabilities in Gas

Turbines and Rocket Engines, May 30 - June 02, 2016 Munich, Germany. 


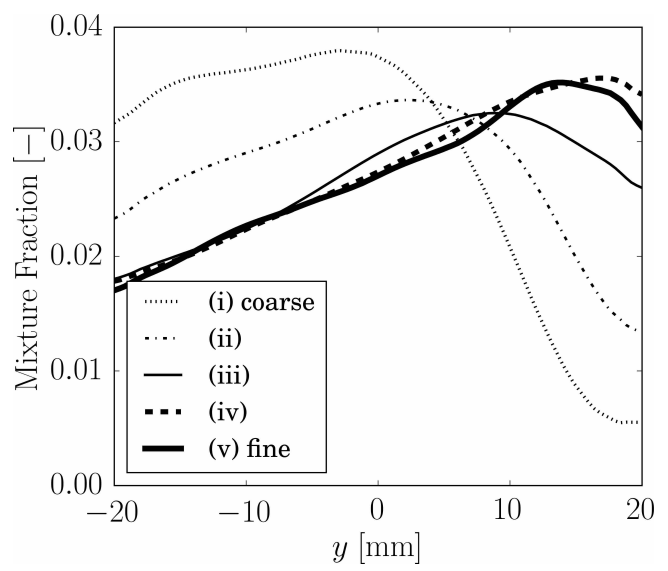

Figure 2: Mesh Study: Profiles of mean Bilger [2] mixture fraction $Z$ at the inlet of the combustion chamber for five different cell sizes.

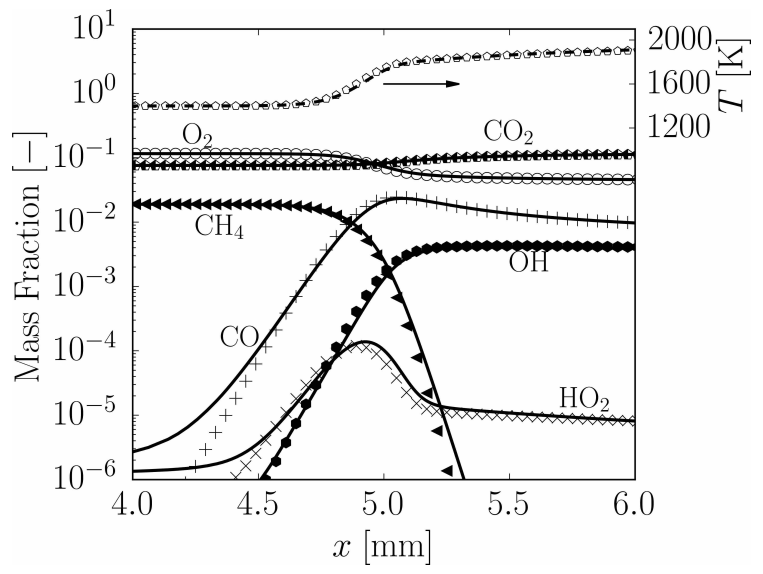

Figure 3: Profiles of mass fractions and temperature of a 1-D propagating flame at equivalence ratio $\phi=0.65$ (global $\phi$ of this configuration) derived with detailed (lines, CANTERA) and reduced chemistry (symbols, AVBP).

position of the combustion chamber inlet. Note that throughout this study the mixture fraction definition formulated by Bilger [2] is used. For the meshes (i) and (ii) there is a strong mismatch in mixture fraction distribution compared to the finest mesh (v), whereas for mesh (iii) $Z$ is underpredicted only for $y>10 \mathrm{~mm}$. The corresponding cell size of mesh (iv) is considered as sufficiently small for this study.

The Analytically Reduced Chemistry (ARC) mechanism ARC_22_GRI211 derived from the detailed GRI211 mechanism [8] is used. For reduction 9 species were removed from the detailed mechanism and a QuasiSteady State approximation was applied to 18 species giving an analytical expression for their concentrations without solving their transport equation. The remaining 22 transported species are the following: $\mathrm{N}_{2}, \mathrm{H}$, $\mathrm{H}_{2}, \mathrm{O}, \mathrm{O}_{2}, \mathrm{OH}, \mathrm{H}_{2} \mathrm{O}, \mathrm{H}_{2} \mathrm{O}_{2}, \mathrm{HO}_{2}, \mathrm{CO}, \mathrm{CH}_{2} \mathrm{O}, \mathrm{CH}_{3}, \mathrm{CH}_{3} \mathrm{OH}, \mathrm{C}_{2} \mathrm{H}_{2}, \mathrm{CH}_{4}, \mathrm{C}_{2} \mathrm{H}_{6}, \mathrm{C}_{2} \mathrm{H}_{4}, \mathrm{CO}_{2}, \mathrm{NO}, \mathrm{HCN}$, $\mathrm{NO}_{2}$ and $\mathrm{N}_{2} \mathrm{O}$.

One can see in Fig. 3 that using ARC_22_GRI211, species mass fractions show excellent agreement with the detailed scheme. A more comprehensive validation of ARC_22_GRI211 can be found in [22].

For turbulent combustion modelling the Dynamic Thickened Flame (DTF) model [6] resolving the flame front on the LES grid is applied. While maintaining the correct laminar speed, a thickening factor is applied in the flame region, so that a minimum of 4.5 cells are located inside the flame front. For a more detailed description the reader is referred to [6]. Moreover the reader can find an application of the DTF model to

This is a pre-print version. Presented at International Symposium: Thermoacoustic Instabilities in Gas

Turbines and Rocket Engines, May 30 - June 02, 2016 Munich, Germany. 


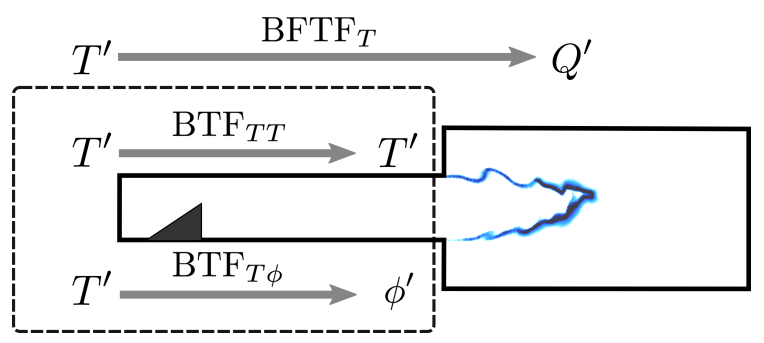

Figure 4: SISO relations to obtain burner flame transfer function $\mathrm{BFTF}_{T}$ with combustor considered as Black Box and burner transfer functions $\mathrm{BTF}_{T T}$ and $\mathrm{BTF}_{T \phi}$ as individual subsystems to gain insight into the Black Box.

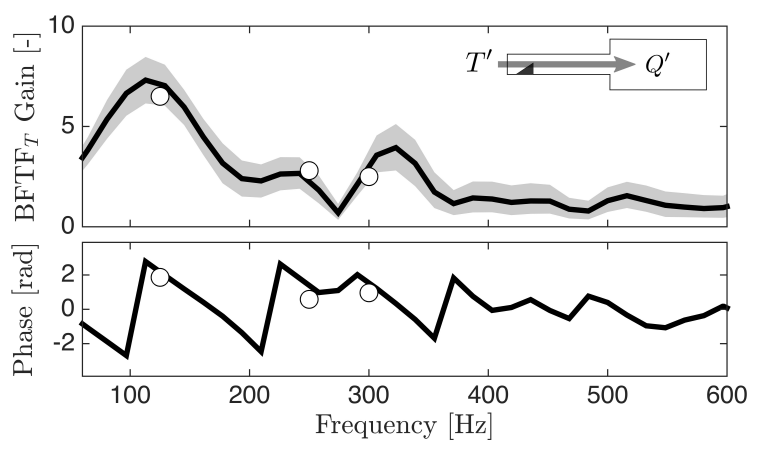

Figure 5: Gain and phase of burner flame transfer function $\left(\mathrm{BFTF}_{T}\right)$ for temperature fluctuations $T^{\prime}$. Excitation amplitude $3 \%$. Symbols derived from single frequency harmonic excitations. $85 \%$ confidence interval in grey.

the Cabra flame configuration showing very good agreement with the experiment, as well as a validation of the DTF model in [22].

\section{Flame response to temperature fluctuations of the sequential burner}

\subsection{Burner flame transer function}

In this part of the section the objective is to determine the flame response, also known as flame transfer function (FTF) of the sequential burner to entropy fluctuations $T^{\prime}$, which are imposed with a broadband excitation signal and subsequently with single frequency harmonic excitation signals at the domain inlet. The combustor is treated as a Black Box with flame and burner, including the two vortex generators, fuel injection and mixing zone. Therefore the flame response to $T^{\prime}$ is called burner flame transfer function:

$$
\operatorname{BFTF}_{T}(\omega)=\frac{Q^{\prime}(\omega) / \bar{Q}}{T^{\prime}(\omega) / \bar{T}},
$$

where $Q$ is the spatially integrated heat release rate. The overbars and primes denote temporal averages and fluctuations expressed as $Q^{\prime}=Q-\bar{Q}$ and $T^{\prime}=T-\bar{T}$. Time signals from broadband excitation are post-processed with the single-input-single-output (SISO) system identification (SI) method and the considered input-output relation of $\mathrm{BFTF}_{T}$ is sketched in the upper part of Fig. 4. Readers should note that throughout the paper extracted temperatures, velocities and equivalence ratios are spatially integrated quantities in a transverse cut.

The simulated physical time to extract the $\mathrm{BFTF}_{T}$ is $N \Delta t=110 \mathrm{~ms}$, with sample length $N$ and sampling increment $\Delta t$, corresponding to 11 flow-through times. As shown in [17] the frequency resolution $\Delta f$, which also represents the lower frequency limit $f_{\min }$, is determined as $\Delta f=f_{\min }=1 / N \Delta t \approx 9 \mathrm{~Hz}$. A best practice

This is a pre-print version. Presented at International Symposium: Thermoacoustic Instabilities in Gas

Turbines and Rocket Engines, May 30 - June 02, 2016 Munich, Germany. 


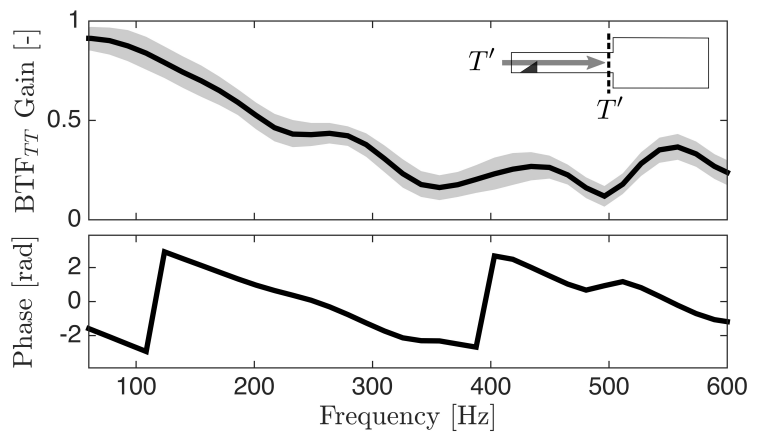

Figure 6: Gain and phase of the burner transfer function $\left(\mathrm{BTF}_{T T}\right)$ relating temperature fluctuations $T^{\prime}$ at the domain inlet to $T^{\prime}$ at the combustion chamber inlet. $85 \%$ confidence interval in grey. Same simulation as in Fig. 5 .

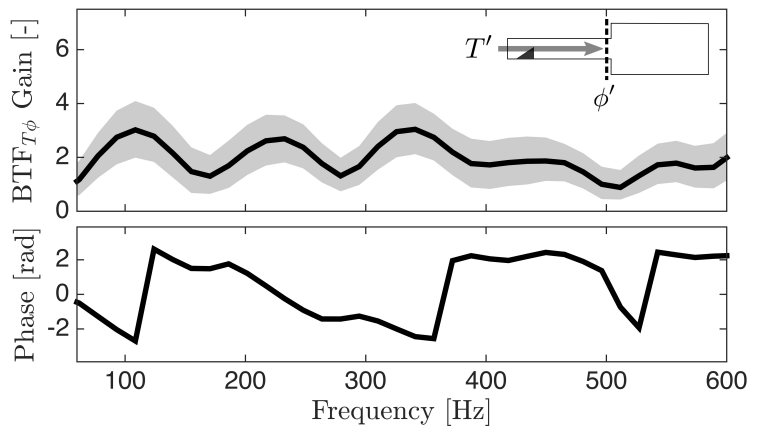

Figure 7: Gain and phase of the burner transfer function $\left(\mathrm{BTF}_{T \phi}\right)$ relating temperature fluctuations $T^{\prime}$ at the domain inlet to equivalence fluctuations $\phi^{\prime}$ at the combustion chamber inlet. $85 \%$ confidence interval in grey. Same simulation as in Fig. 5.

recommendation given in [17] states that for system identification (SI) the filter order $L$ should be chosen such that the filter memory $L \Delta t$ is not smaller than any relevant time lag of the Black Box. By using $L=35$ which gives $L \Delta t=17.5 \times 10^{3} \mathrm{~s}$, and by defining the biggest relevant time lag as the convection time from the domain inlet to the flame front $\Delta x / u_{H G}=7.7 \times 10^{3} \mathrm{~s}$, this recommendation is satisfied.

$\mathrm{BFTF}_{T}$ gain and phase for a temperature excitation amplitude of $3 \%$ are shown in Fig. 5. A region of high gains is present in the frequency range between $80 \mathrm{~Hz}$ to $130 \mathrm{~Hz}$. Note that there is a minimum of gain at $280 \mathrm{~Hz}$ surrounded by two local maxima. The $\mathrm{BFTF}_{T}$ shows very good agreement with the results from single frequency harmonic excitations at the same amplitude for phase and gain.

\subsection{Burner transfer functions}

The Black Box model approach gives only very limited insight into the $\mathrm{BFTF}_{T}$ and therefore the relationship between temperature perturbations imposed at the inlet and (i) resulting temperature fluctuations $T^{\prime}$, as well as (ii) induced equivalence ratio fluctuations $\phi^{\prime}$ at the end of the mixing section are now considered. The input-output relations are expressed via the burner transfer functions $\mathrm{BTF}_{T T}$ and $\mathrm{BTF}_{T \phi}$ (see bottom sketch in Fig. 4).

The reader should be aware that autoignition kernels are observed in the downstream end of the mixing section for the considered operating condition and imposed excitation. Therefore, the output quantities used for the SISO identification of the BTF subsystems are extracted $5 \mathrm{~cm}$ upstream of the mixing section exit.

Gain and phase of the burner transfer function $\mathrm{BTF}_{T T}$ are shown in Fig. 6, with data obtained from the same simulation as in Fig. 5. The burner exhibits a low pass filter behaviour for convected $T^{\prime}$. Hence entropy waves reaching the flame are characterised by a strongly attenuated high frequency content due to

This is a pre-print version. Presented at International Symposium: Thermoacoustic Instabilities in Gas

Turbines and Rocket Engines, May 30 - June 02, 2016 Munich, Germany. 
the low-pass filter nature of the burner.

A modulation of temperature at the hot-gas inlet induces a modulation of density $\rho_{H G}$. This results into fluctuations of the momentum flux ratio $J$ of the fuel jet into the hot-gas cross flow:

$$
J^{\prime}=\frac{\rho_{j e t} \cdot u_{j e t}^{2}}{\rho_{H G}^{\prime} \cdot u_{H G}^{2}} .
$$

Species mixing in jet in cross flow configurations is strongly influenced by $J$ (e.g. [24]) and therefore $\phi^{\prime}$ are induced. Gain and phase of $\mathrm{BTF}_{T \phi}$ are presented in Fig. 7. It is shown that temperature perturbations induce $\phi^{\prime}$ over the whole frequency range with a decrease of gains for frequencies higher than $400 \mathrm{~Hz}$. Here it is worth pointing out that a local maxima at $120 \mathrm{~Hz}$, as well as a local minima at $280 \mathrm{~Hz}$ surrounded by two local maxima can be observed. The same maxima and minima at the same frequencies are present in the $\mathrm{BFTF}_{T}$ in Fig. 5. This may be attributed to constructive or destructive interaction between heat release rate response respectively associated with temperature fluctuation induced $\phi^{\prime}$ and $T^{\prime}$.

\subsection{Flame analysis of harmonic temperature excitation simulation}

Phase-averaged heat release rates $\hat{Q}$ and instantaneous heat release rates $Q$ for one oscillation cycle obtained by a harmonic excitation simulation at $125 \mathrm{~Hz}$ are presented in Fig. 8. For the phase-averaging 8 oscillation cycles were post-processed. As observed from the time signals (not presented in this study) maximum heat release rate response $Q^{\prime}$ occurs at $\pi / 2$ and minimum $Q^{\prime}$ at $3 \pi / 2$. The phase-average results show that during the oscillation cycle phase-averaged heat release rates $\hat{Q}$ distributions exhibit strong spatial variations for low $\hat{Q}$ (coloured in grey and black), whereas the position of the high $\hat{Q}$ reaction zone (in blue) is not significantly changing. For example, for $3 \pi / 2$ no $\hat{Q}$ is present upstream of the flame, whereas for $\pi / 2$ low $\hat{Q}$ already occur in the mixing zone and induce an earlier increase of temperature as visualised by the yellow isolines. In the instantaneous results localised $Q$ spots inside the mixing zone and downstream of the combustion chamber inlet are observed at $2 \pi$ and $\pi / 2$. These spots can evolve into a local flamefront close to the combustion chamber inlet with significant $Q$, as seen at $\pi / 2$.

\subsection{Summary and discussion}

The results presented in Fig. 5 to 8 are summarised and discussed in the following. It is shown that the $\mathrm{BFTF}_{T}$ exhibits high gains at low frequencies, ranging between $80 \mathrm{~Hz}$ and $130 \mathrm{~Hz}$. As seen in the $\mathrm{BTF}_{T T}$ in that frequency range entropy waves reach the flame nearly without attenuation due to the low-pass filter characteristics of the burner. It is also shown that temperature fluctuations upstream of the fuel injection induce $\phi^{\prime}$ and that the $\mathrm{BTF}_{T \phi}$ has a strong peak in gain in the considered frequency range. These observations indicate that strong heat release response $Q^{\prime}$ occur when convected temperature fluctuation induced $T^{\prime}$ and $\phi^{\prime}$ have high oscillation amplitudes and vice versa for example seen at $280 \mathrm{~Hz}$. A summary is given with two extrema highlighted: Fig. 9a, high amplitudes of induced $T^{\prime}$ and $\phi^{\prime}$ yielding in strong heat release
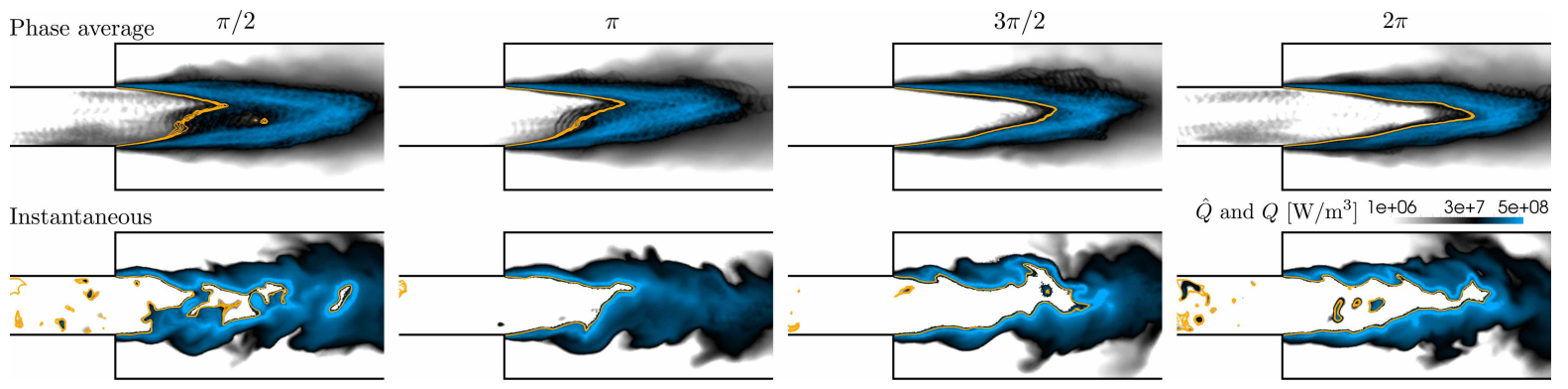

Figure 8: Phase-averaged heat release rates $\hat{Q}$ (top) and instantaneous heat release rates $Q$ (bottom) during one oscillation cycle from $\pi / 2$ to $2 \pi$ (from left to right) for a single frequency harmonic excitation at $125 \mathrm{~Hz}$. $\pi / 2$ correspond to maximum heat release rate oscillations $Q^{\prime}$ and $3 \pi / 2$ to minimum $Q^{\prime}$. Isolines at $1390 \mathrm{~K} \leq T \leq 1410 \mathrm{~K}$ in yellow.

This is a pre-print version. Presented at International Symposium: Thermoacoustic Instabilities in Gas Turbines and Rocket Engines, May 30 - June 02, 2016 Munich, Germany. 
(a)
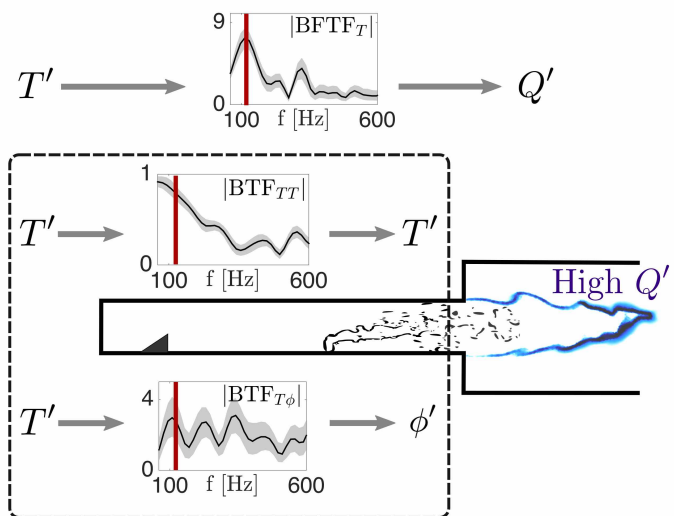

(b)
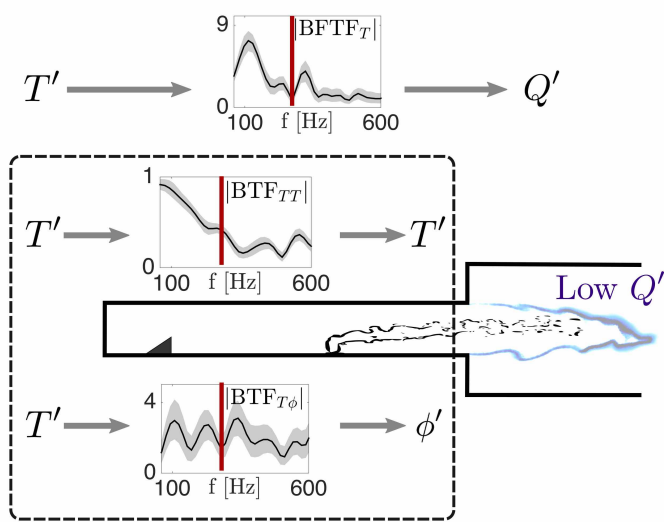

Figure 9: Non-linear flame response in present sequential reheat burner configuration. Two extrema are highlighted: (a) high amplitudes of induced temperature oscillations $T^{\prime}$ and high amplitudes of induced equivalence ratio fluctuations $\phi^{\prime}$ yielding in strong heat release rate oscillations $Q^{\prime}$ and (b) Low amplitudes of $T^{\prime}$ and low amplitudes of $\phi^{\prime}$ resulting in low $Q^{\prime}$. BFTF ${ }_{T}$ burner flame transfer function for $T^{\prime} . \mathrm{BTF}_{T T}$ and $\mathrm{BTF}_{T \phi}$ : burner transfer function that relate $T^{\prime}$ at the domain inlet to $T^{\prime}$ and $\phi^{\prime}$ at the mixing section outlet (compare with Fig. 4).

rate oscillations $Q^{\prime}$ and Fig. 9b, low amplitudes of induced $T^{\prime}$ and $\phi^{\prime}$ resulting in low $Q^{\prime}$.

The instantaneous and phase-averaged results in such a extreme case with a frequency corresponding to Fig. 9a and therefore to high $Q^{\prime}$, indicate that at one instant in the oscillation cycle the combination of high $T^{\prime}$ and $\phi^{\prime}$ can result in a locally strong decrease of autoignition times and auto-igniting spots can occur. This is observed when high temperature stratifications propagate through the mixing zone toward the flame. As shown for example in [22] localised autoignition spots initiate diffusion of heat toward richer mixture fractions and eventually become propagating type flames with significantly high heat release rates, which is observed in the instantaneous flow fields (see Fig. 8 at $\pi / 2$ ). One could assume that this constructive interaction would be enhanced at maximum $T^{\prime}$ with a instantaneous positive amplitude in combination with maximum $\phi^{\prime}$ and a instantaneous negative amplitude (phase shift of $\pi$ ), which is not further investigated in the present study. In contrast, when low temperature stratifications are convected toward the flame, barely any autoignition kernels are observed (see Fig. 8 at $3 \pi / 2$ ).

Note that the analysis is done using the equivalence ratio $\phi$. The above discussion shows that local autoignition plays an important role in the flame response. Therefore the mixture fraction $Z$ is a more convenient quantity for future analysis. Both $\phi$ and $Z$ can be expressed via the other.

\section{Linear and non-linear flame response in a simplified configuration}

In the previous section it is shown that more insight into the burner flame transfer function (BFTF) is gained by relating temperature perturbations imposed at the inlet to induced $T^{\prime}$ and induced equivalence ratio fluctuations $\phi^{\prime}$ upstream of the flame. As a next step the respective contributions of different types of perturbations is investigated, with the aim to break down the flame response of the technically premixed configuration of section 4 into independent driving mechanism and investigate the start of non-linear flame response to these perturbations.

The following results are derived in perfectly premixed conditions with $\phi=0.65$. The inlet temperature is increased to $1490 \mathrm{~K}$ which leads to a comparable flame length as for technically premixed case. The computational mesh ( 0.5 million nodes) without vortex generators and fuel injection is shown in Fig. 10. Heights and widths are the same as in the technically premixed configuration (see Fig. 1a).

Note that the aim of the perfectly premixed configuration is not a representation of the technically premixed flame, it is rather a simplified configuration to identify the respective contributions of different types of perturbations and the onset of the non-linear flame response in a pure and computationally less demanding

This is a pre-print version. Presented at International Symposium: Thermoacoustic Instabilities in Gas

Turbines and Rocket Engines, May 30 - June 02, 2016 Munich, Germany. 


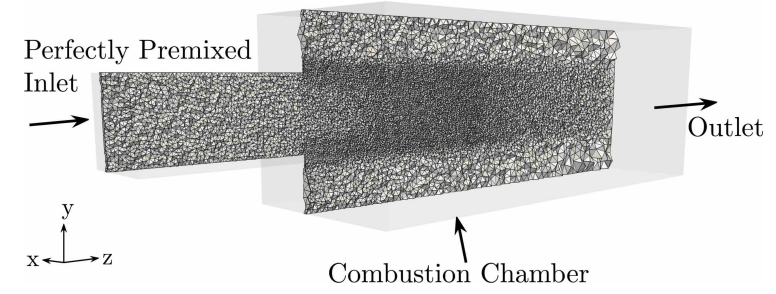

Figure 10: Mesh center cut of perfectly premixed domain. Distance inlet to combustion chamber: $100 \mathrm{~mm}$. Length of combustion chamber: $280 \mathrm{~mm}$.
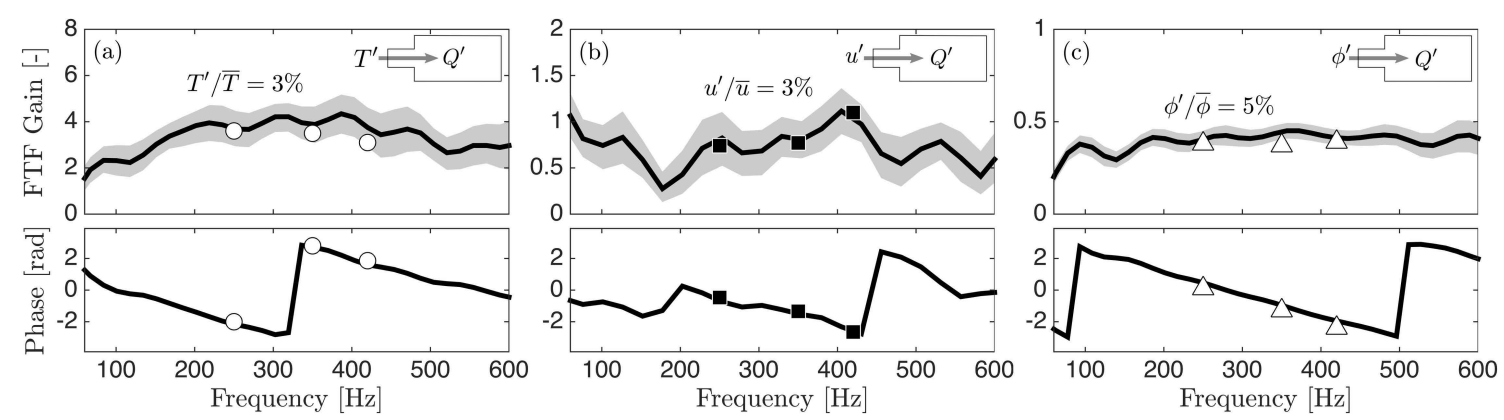

Figure 11: FTF gain and phase for temperature fluctuations $T^{\prime}$, velocity fluctuations $u^{\prime}$ and equivalence ratio fluctuations $\phi^{\prime}$ in perfectly premixed conditions. Symbols show simulations with single frequency harmonic excitations. $85 \%$ confidence interval in grey.

setting.

The linear and non-linear flame frequency responses to flow modulations imposed with broadband signals and subsequently with harmonic excitation signals at the domain inlet are derived. One defines three transfer functions respectively relating temperature $T$, equivalence ratio $\phi$ and velocity $u$ input to the heat release rate $Q$ output:

$$
\begin{aligned}
& \operatorname{FTF}_{T}(\omega)=\frac{Q^{\prime}(\omega) / \bar{Q}}{T^{\prime}(\omega) / \bar{T}}, \\
& \operatorname{FTF}_{u}(\omega)=\frac{Q^{\prime}(\omega) / \bar{Q}}{u^{\prime}(\omega) / \bar{u}},
\end{aligned}
$$

and

$$
\operatorname{FTF}_{\phi}(\omega)=\frac{Q^{\prime}(\omega) / \bar{Q}}{\phi^{\prime}(\omega) / \bar{\phi}} .
$$

Based on these flame transfer functions, single frequency excitation simulations with varying amplitudes are conducted to identify the onset of a non-linear flame response for one single frequency.

The flame transfer function for a temperature excitation amplitude of $3 \%$ is presented in Fig. 11a, showing relatively high gains for frequencies between $200 \mathrm{~Hz}$ and $500 \mathrm{~Hz}$. Single frequency harmonic excitations show excellent agreement for gain and phase with the derived $\mathrm{FTF}_{T}$.

The flame response to velocity fluctuations with an amplitude of $3 \%$ as well as single frequency excitations is presented in Fig. 11b. Compared to entropy excitations, $\mathrm{FTF}_{u}$ gains are relatively low over the whole frequency range.

In Fig. 11c the flame transfer function for equivalence ratio fluctuations is presented. They were induced by varying the $\mathrm{O}_{2}$ mass fraction, which is compensated by a varying $\mathrm{N}_{2}$ mass fraction. The total mass flow, as well as the $\mathrm{CH}_{4}$ mass fraction remain constant. $\mathrm{FTF}_{\phi}$ gains over the whole frequency range are lower than 0.5. Note that a single frequency simulation with varying $\mathrm{CH}_{4}$ mass fraction and compensation by $\mathrm{N}_{2}$, as

This is a pre-print version. Presented at International Symposium: Thermoacoustic Instabilities in Gas

Turbines and Rocket Engines, May 30 - June 02, 2016 Munich, Germany. 

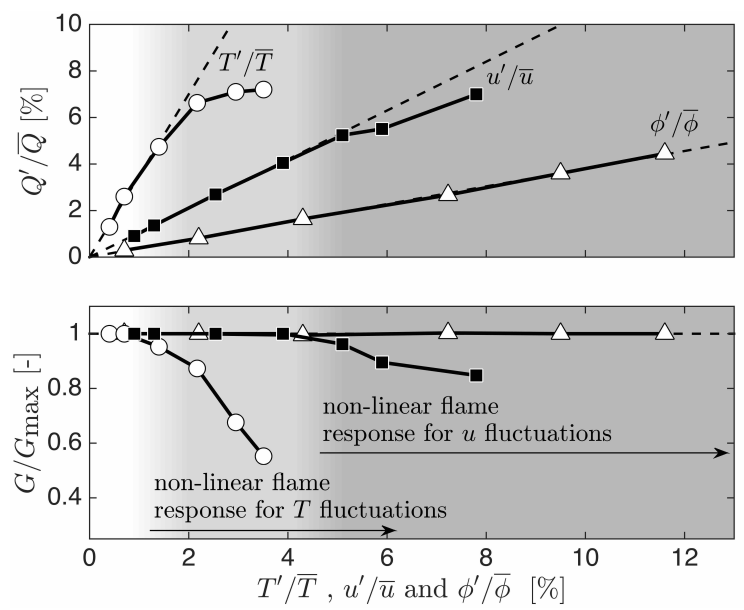

Figure 12: Linear and non-linear flame response for temperature $T^{\prime}$, velocity $u^{\prime}$ and equivalence ratio fluctuations $\phi^{\prime}$ at a constant frequency of $420 \mathrm{~Hz}$. Dotted lines correspond to linear flame responses. Upper plot: Heat release rate fluctuations $Q^{\prime}$ normalised by average heat release rate $\bar{Q}$. Bottom plot: Transfer Function Gain $G$ normalised by maximum gain $G_{\max }$.

done in [3], was performed. The gain at $420 \mathrm{~Hz}$ was shifted from 0.4 to 0.5 . In a technically premixed case the mixture fraction $Z$ varies and therefore both $\mathrm{CH}_{4}$ and $\mathrm{O}_{2}$ would be modified. Nevertheless only varying the $\mathrm{O}_{2}$ mass fraction was considered acceptable for the purpose of the present study.

The start of non-linear behaviour for the three different types of modulation with harmonic perturbation at $420 \mathrm{~Hz}$ is shown in Fig. 12. The non-linear response for $u^{\prime}$ starts at amplitudes ranging between $4 \%$ and $5 \%$. In contrast, for $T^{\prime}$ non-linear behaviour is observed for relatively low amplitudes starting from $1 \%$, which corresponds to an oscillation amplitude of $T^{\prime}=15 \mathrm{~K}$. In the considered range a non-linear behaviour for $\phi^{\prime}$ was not observed up to an amplitude of $12 \%$ of the mean equivalence ratio.

Summarising these results, there is a strong nonlinearity of the flame response to $T^{\prime}$ starting from an amplitude of $1 \%$ of the mean temperature. It is important to note that these results are obtained from harmonic excitation at $420 \mathrm{~Hz}$. Therefore, further investigation are required to fully characterise the nonlinear frequency-dependent heat release rate response in this premixed case.

Considering also the results of section 4 it is shown that the obtained $\mathrm{FTF}_{T}$ gains for $T^{\prime}$ for example in the frequency range between $80 \mathrm{~Hz}$ and $130 \mathrm{~Hz}$ are approximately three times smaller than in the technically premixed $\mathrm{BFTF}_{T}$ (see Fig. 5). One can assume that the separated independent subsystems play a minor role in the global flame response and that rather combined $\phi^{\prime}$ and $T^{\prime}$ strongly affect the flame response as shown in section 4 . This emphasises the strong non-linearity of the technically premixed system and highlights the difficulty to break down the global flame response into independent driving mechanisms using such a "simplified" configuration.

\section{Summary and Outlook}

In the present paper the heat release response of a sequential combustor flame to temperature perturbations is investigated. The combustor is considered as a Black Box and the flame response is given in the burner flame transfer function (BFTF), obtained by using a broadband-excitation based system identification (SI) approach and harmonic single frequency excitations. With the aim to gain more insight into the Black Box, two burner transfer functions (BTF) are obtained. The BTF relate entropy fluctuations imposed at the inlet to induced (i) $T^{\prime}$ and (ii) $\phi^{\prime}$, both extracted at the end of the mixing section. Another goal is to quantify the respective contributions of different types of perturbations to the global flame response and therefore a simplified configuration with perfectly premixed conditions is considered. The main findings in the paper are:

This is a pre-print version. Presented at International Symposium: Thermoacoustic Instabilities in Gas Turbines and Rocket Engines, May 30 - June 02, 2016 Munich, Germany. 
1. The flame response to $T^{\prime}$ starts to be nonlinear for low amplitudes of $T^{\prime}$. In this study nonlinearity was observed for an amplitude of $1 \%$ of the mean temperature.

2. Temperature excitation upstream of the fuel injection induce mixture fraction oscillations $Z^{\prime}$ (and therefore $\phi^{\prime}$ ) which are convected toward the flame.

3. The combination of $T^{\prime}$ and $Z^{\prime}$ yields a highly nonlinear heat release rate response $Q^{\prime}$. Maximum gains of the burner flame transfer function (BFTF) with respect to inlet temperature excitations are observed at a combination of high amplitudes of induced $T^{\prime}$ and $Z^{\prime}$ upstream of the reaction zone.

4. Instantaneous and phase-averaged flow fields in a harmonic $T^{\prime}$ simulation at a frequency corresponding to such high $Q^{\prime}$, show local autoignition kernels inside the mixing zone at an instant of time when maximum temperatures are present. These auto-igniting spots can evolve into a local flame front with high heat release rates. In contrast, barely any auto-igniting kernels are observed with low temperature stratifications propagating toward the flame.

5. $T^{\prime}$ reaching the flame show a strongly attenuated high frequency content due to the low-pass filter nature of the burner which includes vortex generators, fuel injection and mixing section.

6. It is very difficult to break down the global flame response into independent sub-mechanisms by making use of the here presented "simplified" configuration with perfectly premixed conditions.

For future work this paper points out that there is a very strong need of methods to perform non-linear system identification to obtain the non-linear sequential combustor flame response to flow perturbations, especially as shown in this study for temperature fluctuations. Although harmonic excitations show good agreement with the flame response derived from CFD/SI, the reader should be aware that the CFD/SI approach assumes a linear, time-invariant (LTI) system, which as shown in this study is not given even for small levels of temperature oscillations starting from $1 \%$ of the mean temperature.

\section{Acknowledgement}

This research is supported by the Swiss National Science Foundation under Grant 160579.

\section{References}

[1] Ahrens, D., Kolb, M., Hirsch, C., and Sattelmayer, T. (2014). NOx formation in a reacting premixed jet in hot cross flow. In ASME Turbo Expo.

[2] Bilger, R. W., Starner, S. H., and Kee, R. J. (1990). On reduced mechanisms for methane-air combustion in nonpremixed flames. Combust. Flame, 80(2).

[3] Birbaud, A. L., Ducruix, S., Durox, D., and Candel, S. (2008). The nonlinear response of inverted "V" flames to equivalence ratio nonuniformities. Combust. Flame, 154(3).

[4] Cabra, R., Chen, J. Y., Dibble, R. W., Karpetis, a. N., and Barlow, R. S. (2005). Lifted methane-air jet flames in a vitiated coflow. Combust. Flame, 143(4).

[5] Candel, S., Durox, D., Schuller, T., Bourgouin, J.-F., and Moeck, J. P. (2014). Dynamics of swirling flames. Annu. Rev. Fluid Mech., 46.

[6] Colin, O., Ducros, F., Veynante, D., and Poinsot, T. (2000). A thickened flame model for large eddy simulations of turbulent premixed combustion. Phys. Fluids, 12(7).

[7] Ćosić, B., Terhaar, S., Moeck, J. P., and Paschereit, C. O. (2014). Response of a swirl-stabilized flame to simultaneous perturbations in equivalence ratio and velocity at high oscillation amplitudes. Combust. Flame, 162.

[8] C.T. Bowman, R.K. Hanson, D.F. Davidson, W.C. Gardiner, Jr., V. Lissianski, G.P. Smith, D.M. Golden, M. Frenklach and M. Goldenberg (http://www.me.berkeley.edu/gri_mech/).

[9] Ghani, A., Poinsot, T., Gicquel, L., and Müller, J.-D. (2016). LES Study of Transverse Acoustic Instabilities in a Swirled Kerosene/Air Combustion Chamber. Flow, Turbul. Combust., 96.

[10] Gicquel, L. Y. M., Gourdain, N., Boussuge, J. F., Deniau, H., Staffelbach, G., Wolf, P., and Poinsot, T. (2011). High performance parallel computing of flows in complex geometries. Comptes Rendus - Mec., 339(2-3).

[11] Güthe, F., Hellat, J., and Flohr, P. (2009). The Reheat Concept: The Proven Pathway to Ultralow Emissions and High Efficiency and Flexibility. J. Eng. Gas Turbines Power, 131(2).

[12] Hermeth, S., Staffelbach, G., Gicquel, L. Y. M., Anisimov, V., Cirigliano, C., and Poinsot, T. (2014). Bistable swirled flames and influence on flame transfer functions. Combust. Flame, 161(1).

[13] Jangi, M., Zhao, X., Haworth, D. C., and Bai, X.-S. (2015). Stabilization and liftoff length of a non-premixed methane/air jet flame discharging into a high-temperature environment: An accelerated transported PDF method. Combust. Flame, $162(2)$.

This is a pre-print version. Presented at International Symposium: Thermoacoustic Instabilities in Gas Turbines and Rocket Engines, May 30 - June 02, 2016 Munich, Germany. 
[14] Lieuwen, T. C. and Zinn, B. T. (2005). Combustion Instabilities: Basic Concepts.

15] Mastorakos, E. (2009). Ignition of turbulent non-premixed flames. Prog. Energy Combust. Sci., 35(1).

[16] Oberleithner, K., Schimek, S., and Paschereit, C. O. (2015). Shear flow instabilities in swirl-stabilized combustors and their impact on the amplitude dependent flame response: A linear stability analysis. Combust. Flame, 162(1).

[17] Polifke, W. (2014). Black-box system identification for reduced order model construction. Ann. Nucl. Energy, 67.

[18] Prathap, C., Galeazzo, F. C. C., Kasabov, P., Habisreuther, P., Zarzalis, N., Beck, C., Krebs, W., and Wegner, B. (2012). Analysis of NOX Formation in an Axially Staged Combustion System at Elevated Pressure Conditions. J. Eng. Gas Turbines Power, 134(3).

[19] Prause, J., Noll, B., Aigner, M., and Syed, K. (2014). Sensitivity Analysis of Autoignition Simulation At Gas Turbine Operating Conditions. In ASME Turbo Expo.

[20] Rayleigh, J. W. S. B. (1878). The Explanation of Certain Acoustical Phenomena. Nature, 18(455).

[21] Roediger, T., Lammel, O., Aigner, M., Beck, C., and Krebs, W. (2013). Part-load operation of a piloted FLOX® combustion system. J. Eng. Gas Turbines Power, 135(3).

[22] Schulz, O., Jaravel, T., Poinsot, T., Cuenot, B., and Noiray, N. (2016 (to be published)). A criterion to distinguish autoignition and propagation applied to a lifted methane-air jet flame. Proc. Combust. Inst., 36.

[23] Silva, C. F., Emmert, T., Jaensch, S., and Polifke, W. (2015). Numerical study on intrinsic thermoacoustic instability of a laminar premixed flame. Combust. Flame, 162(9).

24] Smith, S. H. and Mungal, M. G. (1998). Mixing, structure and scaling of the jet in crossflow. J. Fluid Mech., 357.

[25] Sullivan, R., Wilde, B., Noble, D. R., Seitzman, J. M., and Lieuwen, T. C. (2014). Time-averaged characteristics of a reacting fuel jet in vitiated cross-flow. Combust. Flame, 161(7).

[26] Taamallah, S., Shanbhogue, S. J., and Ghoniem, A. F. (2016). Turbulent flame stabilization modes in premixed swirl combustion: Physical mechanism and Karlovitz number-based criterion. Combust. Flame, 166.

[27] Tautschnig, G., Haner, E., Hirsch, C., and Sattelmayer, T. (2014). Experimental and numerical investigation of confined jets in how co-flow. In ASME Turbo Expo.

[28] Yang, Y., Noiray, N., Scarpato, A., Schulz, O., Düsing, K. M., and Bothien, M. (2015). Numerical analysis of the dynamic flame reponse in Alstom reheat combustion systems. In ASME Turbo Expo.

[29] Zellhuber, M., Schwing, J., Schuermans, B., Sattelmayer, T., and Polifke, W. (2014). Experimental and numerical investigation of thermoacoustic sources related to high-frequency instabilities. Int. J. Spray Combust. Dyn., 6(1). 